\title{
Abdominopelvic Sarcoma of Perivascular Epithelioid Cells. Report of Four Cases in Young Women, One with Tuberous Sclerosis
}

Franco Bonetti, M.D., Guido Martignoni, M.D., Chiara Colato, M.D., Erminia Manfrin, M.D., Marcello Gambacorta, M.D., Maurizio Faleri, M.D., Carlos Bacchi, M.D., Vai-Chong Sin, MBBS, Nim-Lai Wong, M.D., Mark Coady, M.D., John Chan Kwok-cheung, M.D.

Istituto di Anatomia Patologica (FB, GM, EM, CC), Università di Verona; Servizio di Anatomia Patologica (MG, MF), Ospedale Niguarda, Milano, Italy; Department of Pathology (CB), Facultade Medicina-UNESP Botucatu, Brasil; Department of Pathology and Clinical Oncology (VCS, JKCC), Queen Elizabeth Hospital, Hong Kong; Department of Anatomical Pathology (MC), SEALS Prince of Wales Hospital, Sidney, Australia; and Department of Pathology (NLW), Kinag Wu Hospital, Macau

The perivascular epithelioid cell has been proposed to be the unifying proliferating cell type in a number of lesions such as angiomyolipoma, lymphangiomyomatosis, clear cell "sugar" tumor and renal capsuloma. With the exception of rare examples of angiomyolipoma, they are nonmetastasizing. We report four examples of a new member of this family of perivascular epithelioid cell neoplasms that occur in abdominopelvic location and show metastatic properties. The patients, all women, were aged 19 to 41 years (mean, 32), and presented with a tumor mass involving the serosa of the ileum, uterus or pelvic cavity. Morphologically, the tumors were composed of sheets of large polygonal cells with glycogen-rich clear or eosinophilic cytoplasm and moderately pleomorphic nuclei, traversed by a delicate vasculature, mimicking clear cell carcinoma. There were areas of coagulative necrosis and occasional mitotic figures. Intracytoplasmic brown pigment was present in two cases. Spindly cells, smooth muscle and fat were absent. Lymphovascular invasion was present in all, lymph node metastasis was documented in two and metastasis to the ovary was present in one case. Two patients developed widespread metastatic disease after 10 and 28 months from diagnosis. One patient showed the clinical signs of tuberous sclerosis. In

Work supported by Progetto Sanità 96-97 Fondazione Cassa di Risparmio di VR-VI-BL-AN, and Progetti Finalizzati Regione Veneto.

This work was presented in part at the US \& Canadian Academy of Pathology Annual Scientific Meeting, San Francisco, March 1999. Copyright (C) 2001 by The United States and Canadian Academy of Pathology, Inc.

VOL. 14, NO. 6, P. 563, 2001 Printed in the U.S.A

Date of acceptance: November 15, 2000.

Address reprint requests to: Franco Bonetti, M.D., Istituto di Anatomia Patologica, Policlinico B. Roma, Verona, 37134 Italy; e-mail: f.bonetti@mail.univr.it; fax: 39-45-8027136. spite of the epithelial-like appearance, the tumor cells were negative for epithelial markers but were strongly positive with the melanogenesis-related marker HMB45. Another melanogenesis marker (MART-1) was positive in two cases. Other markers including S-100 protein, vimentin, muscle-specific actin, desmin and chromogranin $A$ were negative. Thus, these tumors are not readily classifiable in the existing schema of known entities, and show overlapping morpho-phenotypic features of clear cell "sugar" tumor of the lung and epithelioid angiomyolipoma. We consider them as sarcomas composed of a pure population of uncommitted perivascular epithelioid cell, that lack modulation toward smooth muscle or adipose cells.

KEY WORDS: Perivascular epithelioid cell, Angiomyolipoma, Clear cell "sugar" tumor, Melanoma markers, Tuberous sclerosis.

Mod Pathol 2001;14(6):563-568

This report describes four cases of an unusual abdominopelvic tumor, all occurring in young women, and morphologically strongly mimicking clear cell carcinoma. Immunophenotypic analysis shows that these tumors do not display epithelial characteristics, but are rather HMB45 positive and S100 protein and vimentin negative, supporting that they represent a novel member of the perivascular epithelioid cell (PEC) tumor family that has malignant potential (1).

\section{CLINICAL HISTORY}

\section{Case 1}

A 28-year-old Caucasian woman presented with abdominal pain and physical examination was un- 
revealing. Her past health was unremarkable except for appendicectomy at the age of 16 . Workup included ultrasonography and CT scan, both of which revealed a large solid mass involving the caecum and terminal ileum. Intraoperative examination of the uterus, ovaries and adnexa was unremarkable. An extensive metastatic work-up, including bone scan, chest, abdominal, and pelvic CT scan, was negative. The tumor was removed together with the terminal ileum and ascending colon. The resected specimen contained a firm, well encapsulated mass, $9 \mathrm{~cm}$ in greatest diameter, attached to the serosal surface of the terminal ileum. The cut surface was pale yellowish with necrotic areas. Five enlarged mesenteric lymph nodes were found. Nine months after surgery the patient was doing well with no evidence of tumor recurrence, but after 28 months she developed liver insufficiency and shortly after died of multiple hepatic metastases. No autopsy was performed.

Extensive clinico-radiologic investigation, including brain and total body Ct scan, dermatologic, and neurologic examination, did not reveal signs of tuberous sclerosis complex (TSC). She had high school level of education and worked as accountant.

\section{Case 2}

A 19-year-old Chinese woman presented with a 1-year history of vague abdominal pain. Ultrasonogram showed a large mass in the lower uterine segment. With a clinical diagnosis of leiomyoma, laparotomy was performed. At operation, a necrotic tumor $5.5 \mathrm{~cm}$ in size was found in the posterior lower uterine segment, with involvement of the serosa. There was no obvious involvement of the endometrial cavity. Total hysterectomy was performed.

Ultrasonographic examination 1 month after operation showed bilateral pelvic masses. A second laparotomy was performed, revealing bilateral enlarged pelvic lymph nodes, measuring $6 \mathrm{~cm}$ in maximum diameter on each side. Bilateral salpingooophorectomy was performed together with bilateral pelvic lymphadenectomy, inguinal node dissection and removal of a cuff of vagina. Histologic examination revealed tumor involvement of the pelvic and inguinal nodes as well as the vagina.

She was then referred to the Oncology Department for further management. Computed tomography showed vague soft tissue shadow in the pouch of Douglas, suggestive of residual disease. She was given combination chemotherapy including adriamycin and ifosfamide, followed by consolidation radiotherapy. She remained well for 10 months after therapy, and then developed local recurrence, and multiple metastatic deposits in the lungs and the vertebral spine.
The patient was last seen on may 1999, 18 months after surgery, and she was in very poor and frail condition. She had local recurrence, pulmonary metastasis and multiple bone metastases. Unfortunately, we have further lost contact with her.

There were no external stigmata of tuberous sclerosis and CAT scan of the abdomen failed to reveal renal or liver masses. She had secondary school education and worked as a sales girl.

\section{Case 3}

A 40-year-old white woman underwent surgery because of uterine leiomyomas. During the operation, a $2.5 \times 2 \times 1.5 \mathrm{~cm}$ pelvic nodule was accidentally found and thought to represent endometriosis. Grossly the tumor was well delimited and lobulated. Cut surface revealed a solid tan-colored tumor with a granular quality and punctuated with dark brown to black areas. No hemorrhage or cysts were found. Six months after the resection the patient was well and alive.

Unfortunately, the patient was then lost to follow up and no evaluation of subtle findings possibly related to TSC could be evaluated. However, no evident signs of TSC were present at admission.

\section{Case 4}

A 41-year-old woman underwent hysterectomy and bilateral salpingo-oophorectomy for presumed fibroids. A $6 \mathrm{~cm}$ myometrial mass protruded from the serosal surface. Other tumor masses of lesser diameter were evident in the myometrium and in the subserosa. The right ovary showed a nodular tumor mass measuring $2 \mathrm{~cm}$ in diameter with similar features and was confirmed histologically as consistent with a metastatic deposit from the uterine neoplasm. The left ovary and both fallopian tubes were unremarkable. The myometrial and ovarian tumor masses consisted of invasive whitish-gray, solid, somewhat lobulated rather soft neoplasm showing occasional tiny foci of hemorrhage.

The patient was long known to have the stigmata of TSC with multiple bilateral renal angiomyolipomas and characteristic skin manifestations of the disease. She was unemployed at the time of surgery. Six months after surgery the patient is doing well without evidence of recurrent disease.

\section{MATERIALS AND METHODS}

Tissue obtained at the time of surgery was fixed in $10 \%$ buffered formalin and routinely processed for paraffin embedding. Sections were cut with a thickness of 4 to $6 \mu \mathrm{m}$, and stained with hematoxylin and eosin, periodic acid-Schiff (periodic acidSchiff) reagent, with and without diastase pretreat- 
ment, Gordon and Awe reticulin stain, and Fontana-Masson stain for melanin.

Immunocytochemical analysis was carried out on paraffin sections using the alkaline-phosphataseanti-alkaline-phosphatase and/or peroxidase-antiperoxidase techniques. The sections were stained with the primary antibodies listed in Table 1. Appropriate positive and negative controls were performed.

\section{PATHOLOGY}

The histopathologic features of all tumors were similar, and closely mimicked those of clear cell carcinoma of the kidney. The neoplastic cells were arranged in sheets and packets traversed by a delicate vasculature consisting of a rich network of sinusoid-type blood vessels without extravasated red blood cells. Focally, there was a papillary-like pattern or pseudoglandular appearance (Fig. 1, A-B). The tumors comprised a rather uniform population of large polygonal cells with abundant cytoplasm. The tumor cells had a round to oval nucleus, sometimes with a prominent nucleolus, and exhibited mild to moderate nuclear pleomorphism. The cytoplasm was either entirely clear or partially clear but with interspersed fluffy eosinophilic areas. Case 4 was slightly different for a more pronounced eosinophilia of the cytoplasm and presence of only scanty glycogen (Fig. 2, A-B).

Coagulative necrosis was a prominent feature and appeared as multiple foci of variable size and tumor emboli were seen in lymphovascular spaces at the periphery of the tumor in all cases. Abundant brown pigment was evident in the cytoplasm of a fraction of neoplastic cells (about 10\%), as well as in macrophages in Cases 2 and 3. This pigment was positive with Fontana-Masson stain, suggesting its melanin nature (Fig. 2C). No pigment was present in Cases 1 and 4. Only rare mitotic figures could be found. An interspersed component of adipose cells or abnormal thick-walled blood vessels was not identified. The tu-

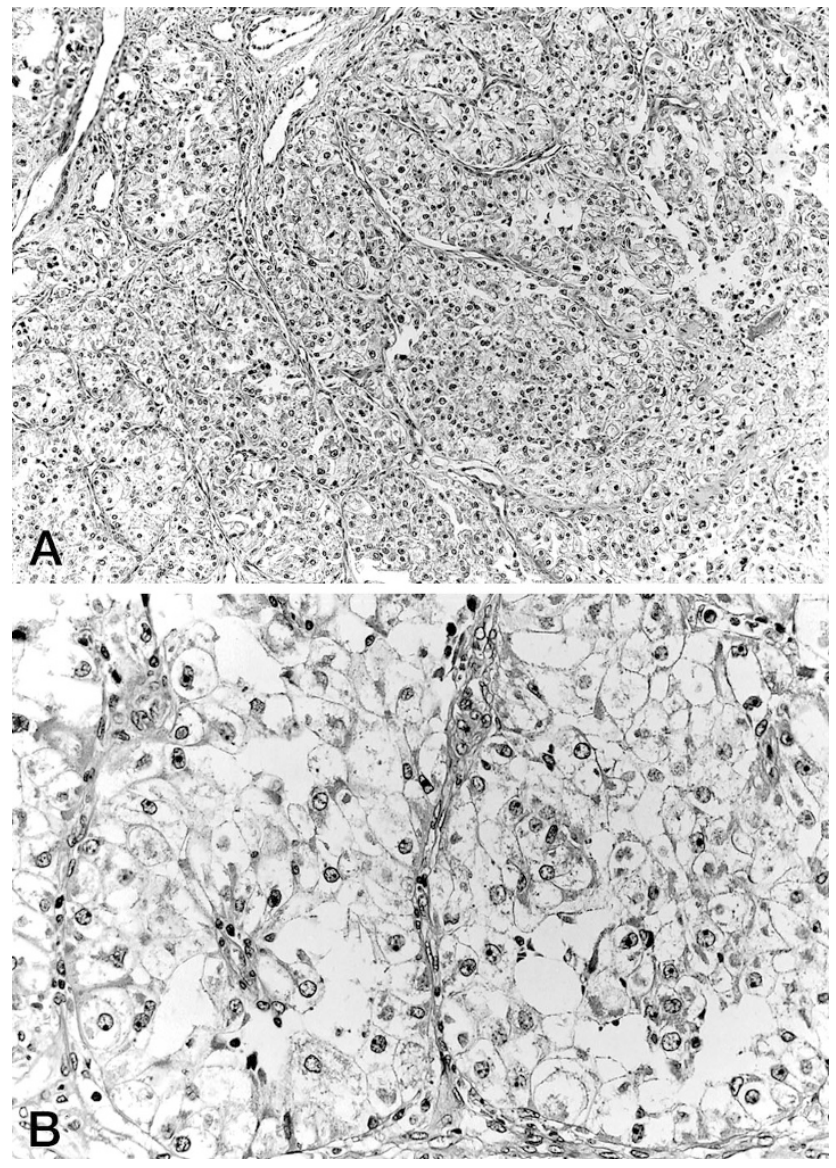

FIGURE 1. A, The large neoplastic cells are arranged in sheets and packets traversed by a delicate vasculature consisting of a rich network of sinusoid-type blood spaces. B, The pseudoglandular appearance combined with the clear cytoplasm of the neoplastic cells results in a strong mimicry of clear cell carcinoma of the kidney.

mor cells contained abundant intracytoplasmic periodic acid-Schiff-positive, diastase-sensitive glycogen. No diastase-resistant periodic acid-Schiff-positive crystalloid structures could be identified. Reticulin fibers surrounded individual cells, and, occasionally, small nests of cells.

TABLE 1. Immunocytochemical Findings in Four Sarcomas of Perivascular Epithelioid Cell

\begin{tabular}{|c|c|c|c|c|c|c|}
\hline Antibody & Source & Dilution & Case 1 & Case 2 & Case 3 & Case 4 \\
\hline CAM 5.2 (Keratin) & Becton-Dickinson, Mountain View, CA & $1: 10$ & Neg & Neg & Neg & Neg \\
\hline AE1 and AE3 (Keratin) & Boehringer, Mannheim, FRG & $1: 500$ & Neg & Neg & Neg & Neg \\
\hline KL1 (Keratin) & Immunotech, Marseille, France & Undiluted & Neg & Neg & Neg & Neg \\
\hline EMA (Epit. membrane Ag) & Dakopatts, Denmark & $1: 500$ & Neg & Neg & Neg & Neg \\
\hline HMB-45 & Dakopatts, Denmark & Undiluted & $80 \%+$ & $90 \%+$ & $60 \%+$ & $80 \%+$ \\
\hline MART-1 & Biogenex, San Roman, CA & $1: 20$ & $80 \%+$ & Neg & Neg & $80 \%+$ \\
\hline S-100 & Dakopatts, Denmark & $1: 500$ & Neg & Neg & Neg & Neg \\
\hline Vimentin & Amersham, Buckinghamshire, England & $1: 10$ & Neg & Neg & Neg & Neg \\
\hline Chromogranin & Cimbus, England & $1: 1000$ & Neg & Neg & Neg & Neg \\
\hline Desmin & Dakopatts, Denmark & $1: 500$ & Neg & Neg & Neg & Neg \\
\hline Muscle specific actin (HHF35) & Ortho-Enzo NY, USA & $1: 1500$ & Neg & Neg & Neg & Neg \\
\hline Estrogen receptor & Dakopatts, Denmark & $1: 50$ & Neg & Neg & Neg & Neg \\
\hline Progesterone receptor & Dakopatts, Denmark & $1: 80$ & $60 \%+$ & Neg & Neg & Neg \\
\hline Smooth muscle actin & Dakopatts, Denmark & $1: 100$ & Neg & Neg & Neg & Neg \\
\hline Mib1 (Ki-67) & Dakopatts, Denmark & $1: 300$ & $2 \%+$ & $2 \%+$ & $4 \%+$ & $5 \%+$ \\
\hline Myosin & Dakopatts, Denmark & $1: 100$ & Neg & Neg & Neg & Neg \\
\hline
\end{tabular}


The lymph nodes in Cases 1 and 2 showed metastatic deposits of the neoplasm (Fig. 3A), whereas Case 4 showed a metastatic deposit in the right ovary.

The immunocytochemical profile of the tumors was similar, and is summarized in Table 1 . The cells were negative for epithelial markers (CAM 5.2, KL1, AE1, AE3, EMA), vimentin, chromogranin-A, S-100 protein (Fig. 2D), desmin, myosin, and musclespecific actin. They showed strong granular staining for the melanogenesis-related markers HMB45 (Fig. 3B) and, in two cases, for MART-1). The Mib1 (Ki-67) was positive in a fraction of the neoplastic cells (range, 2 to $8 \%$ positive).

\section{DISCUSSION}

The tumors described in this report show unusual and contrasting features. The first impression on morphologic analysis is that of a clear cell carcinoma such as that of the kidney or female genital tract. However, the cytoplasm is not generally as water-clear as that commonly seen in renal cell carcinoma, and true glandular formation is lacking.
The focal glandular structures and papillae lined by hobnail cells as seen in clear cell carcinoma of the female genital tract are also absent. These two possibilities are effectively ruled out by the lack of immunohistochemical evidence of epithelial differentiation. A metastasis from unknown primary seems unlikely, as extensive work-up and follow up failed to reveal other primary tumors.

A malignant melanoma is another important consideration because of its ability to mimic epithelial tumors with clear cells such as in clear cell sarcoma of tendons and aponeurosis (melanoma of soft parts) and the presence of melanin pigment in two of four cases. However, the lack of immunoreactivity for S-100 protein and vimentin makes this diagnosis most unlikely (2). In addition, the rich network of reticulum fibers among the tumor cells is rather uncommon in malignant melanoma, where reticulin fibers are usually scanty. Presence of melanin pigment is also not exclusive to melanomas.

Adrenocortical carcinoma, with clear cells, delicate vasculature and A103 (MART-1) immunoreactivity, represents a further differential diagnosis (3). However, they are usually positive, albeit focally, for

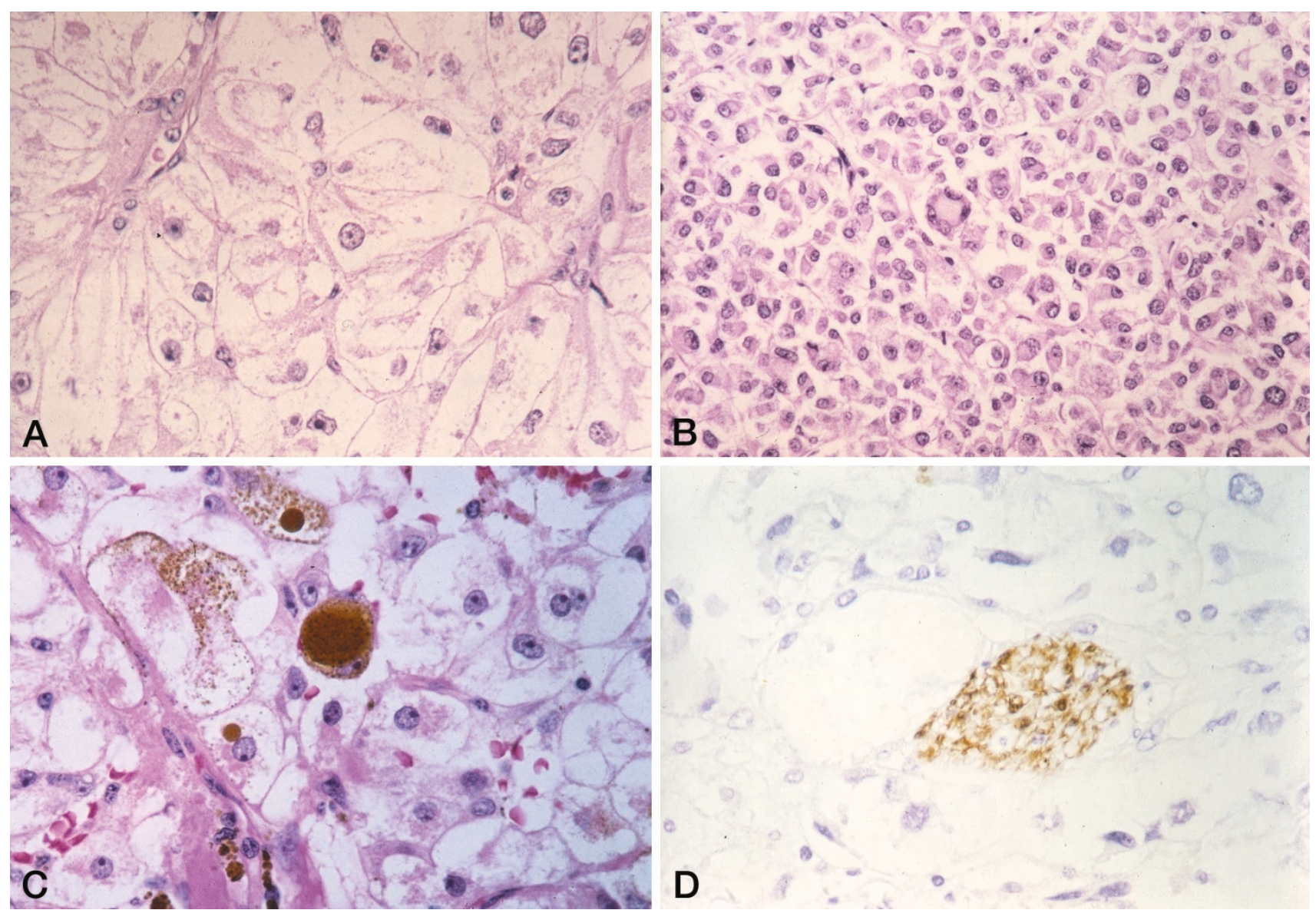

FIGURE 2. A, The tumor cells exhibit a round, often nucleolated, nucleus with abundant cytoplasm, partially clear but with interspersed fluffy eosinophilic areas. B, In other areas, particularly in Case 4, a more pronounced eosinophilia of the cytoplasm can be observed. Occasional multinucleated giant cell are present. C, Abundant brown pigment is evident in the cytoplasm of a fraction of neoplastic cells in Cases 2 and 3. D, the neoplastic cells are S-100 negative and infiltrate a nerve that is S-100 positive. 


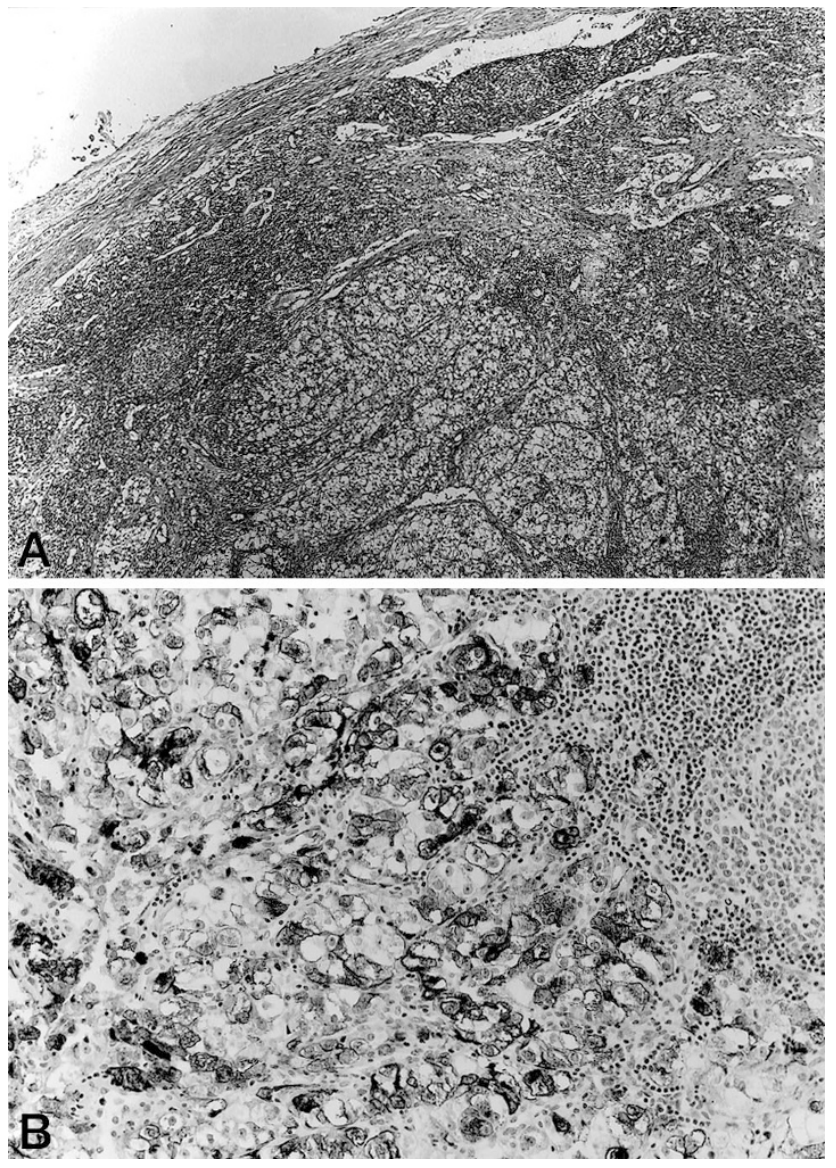

FIGURE 3. A, The lymph nodes in Cases 1 and 2 show metastatic deposits of the neoplasm, and $\mathbf{B}$, the neoplastic cells are strongly positive with HMB45.

cytokeratins and unreactive with HMB45. Pigmented paraganglioma can be excluded on grounds of morphology, HMB45 immunoreactivity, and lack of neuroendocrine marker expression (4, 5).

Thus, the immunohistochemical profile of the tumors reported herein does not exhibit epithelial, endocrine or melanocytic characteristics despite morphologic resemblance to these neoplasms. They show marked histologic and phenotypic similarities with a recently identified family of tumors composed of PECs $(1,6)$ such as the clear cell "sugar" tumor of the lung and monotypic epithelioid angiomyolipoma of the kidney and liver. Recent reports have considerably widened the spectrum of PEC lesions reporting them in the uterus, pancreas and in the falciform ligament (7-11). PEC tumors can mimic renal clear cell carcinoma (1217) epithelioid leiomyosarcoma (18), hepatocellular carcinoma $(19,20)$ conventional leiomyosarcoma or other high grade sarcomas.

The family of PEC tumors can show multiorgan involvement, and at times a distinction between metastatic spread and multifocal disease can be difficult to make. Also, the classical benign AML can present with multiple lesions particularly in the regional lymph nodes and in the contralateral kidney. These have been considered multifocal lesions rather than metastases (21). However, rare cases of malignant AML have been reported, and in one case the metastatic nature of the multiple localizations has been demonstrated with molecular analysis (22).

The present cases differ from these tumors in the clinical presentation, the consistent malignant behavior manifesting as metastasis, the more pronounced epithelial pseudopapillary pattern and the frequent presence of melanin pigment. This latter finding has not been reported in other related tumors such as monotypic epithelioid angiomyolipoma of the kidney (16). Pigmentation is not restricted to melanocytic tumors and has been reported in a variety of non-melanocytic tumors including a unique case of renal cell carcinoma composed of clear cells (23). Davis et al. (24) have reported in abstract form five pigmented clear cell neoplasms of the kidney. These cases show remarkable similarities with our cases, including predominance in young women and HMB45 immunoreactivity. Nonetheless, malignant behavior was not observed in their cases. More recently, a case of malignant pigmented clear cell epithelioid tumor of the kidney has been reported by Ribalta et al. (25) showing marked similarities with our cases. In a study of 30 hepatic angiomyolipomas, Tsui et al. (20) have shown in five cases the presence of a pigment identical to that observed in our cases. Our cases also differ from monotypic epithelioid angiomyolipoma in the monomorphous cellular population; in epithelioid angiomyolipoma, careful search often reveals areas with the typical cellular composition of angiomyolipoma.

Most PEC lesions are related to the inherited syndrome of TSC (26), and one of our cases showed the characteristic stigmata of this syndrome. Thus we believe that this novel tumor should be included among the TSC-associated neoplasms. A morphologically similar lesion, clear cell "sugar" tumor of the lung, was previously considered a lesion unrelated to TSC, but recent data support its inclusion among the TSC-associated lesions $(1,27,28)$. TSC is a tumor syndrome and can manifest with a variety of neoplasms in different organs. The occurrence of a single sporadic lesion, such as angiomyolipoma or lymphangiomyomatosis, in a patient without other signs of TSC is now considered by some authors a "forme fruste" of the disease $(26,29)$. Molecular analysis has demonstrated that both TSCassociated and sporadic lesions can show the same genetic defect, namely the loss of heterozygosity on $16 \mathrm{p} 13$ for the TSC2 locus (30). This genetic defect, however, may represent a somatic mutation in 
these tumors, and does not necessarily indicate a subclinical evidence of TSC.

In conclusion, we have reported four cases of abdominopelvic malignant tumors, not readily classifiable in the existing schema of known entities, which we believe are composed purely of uncommitted PEC, the main distinguishing features being the occurrence in abdominopelvic extrarenal sites in young women, the pure epithelioid population, clear-eosinophilic cytoplasm, frequent pigmentation, and malignant potential. Two of the tumors showed an aggressive behavior and developed multiple metastases within 20 months, and two showed morphologic features suggestive of malignancy. The presence of vascular invasion in all cases, and of lymph node or ovarian metastases in other two cases is in keeping with a malignant behavior. They represent a new TSC-associated lesion with a marked female predominance.

\section{REFERENCES}

1. Bonetti F, Pea M, Martignoni G, Zamboni G, Manfrin E, Colombari R, et al. The perivascular epithelioid cell and related lesions. Adv Anat Pathol 1997;4:343-58.

2. Swanson PE, Wick MR. Clear cell sarcoma. An immunohistochemical analysis of six cases and comparison with other epithelioid neoplasms of soft tissue. Arch Pathol Lab Med 1989;113:55-60.

3. Busam KJ, Iversen K, Coplan KA, Old LJ, Stockert E, Chen $\mathrm{Y}-\mathrm{T}$, et al. Immunoreactivity for A103, an Antibody to Melan-A (Mart-1), in adrenocortical and other steroid tumors. Am J Surg Pathol 1998;22:57-63.

4. Tavassoli FA. Melanotic paraganglioma of the uterus. Cancer 1986;58:942-8.

5. Chetty R, Clark SP, Taylor DA. Pigmented pheochromocytoma of the adrenal medulla. Hum Pathol 1993;24:420-3.

6. Bonetti F, Pea M, Martignoni G, Doglioni C, Zamboni G, Capelli P, et al. Clear cell ("sugar") tumor of the lung is a lesion strictly related to angiomyolipoma. The concept of a family of lesions characterized by the presence of the perivascular epithelioid cell (PEC). Pathology 1994;26:230-6.

7. Pea M, Martignoni G, Zamboni G, Bonetti F. Perivascular Epithelioid Cell [letter]. Am J Surg Pathol 1996;20:1149-55.

8. Zamboni G, Pea M, Martignoni G, Zancanaro C, Faccioli G, Giglioli E, et al. Clear cell "sugar" tumor of the pancreas. A novel member of the family of lesions characterized by the presence of perivascular epithelioid cells (PEC). Am J Surg Pathol 1996;20:722-30.

9. Folpe AL, Goodman ZD, Ishak KG, Paulino AF, Taboada EM, Meehan SA, et al. Clear cell myomelanocytic tumor of the falciform ligament/ligamentum teres: a novel member of the perivascular epithelioid clear cell family of tumors with a predilection for children and young adults. Am J Surg Pathol 2000;24:1239-46.

10. Tanaka $\mathrm{Y}$, Ijiri R, Kato K, Kato Y, Misugi K, Nakatani $\mathrm{Y}$, et al. HMB-45/melan-A and smooth muscle actine-positive clear cell epithelioid tumor arising in the ligamentum teres hepatis: additional example of clear cell "sugar" tumors. Am J Surg Pathol 2000;24:1295-9.

11. D’Andrea V, Lippolis G, Biancari F, Ruco LP, Marzullo A, Wedar A, et al. A uterine pecoma: a case report. G. Chir 1999;20:163-4.

12. Pea M, Bonetti F, Zamboni G, Martignoni G, Riva M, Colombari $\mathrm{R}$, et al. Melanocyte marker HMB-45 is regularly ex- pressed in angiomyolipoma of the kidney. Pathology 1991; 23:185-8.

13. Mai KT, Perkins DG, Collins JP. Epithelioid cell variant of renal angiomyolipoma. Histopathology 1996;28:277-80.

14. Bjornsson J, Short MP, Kwiatkowski DJ, Henske EP. Tuberoussclerosis-associated renal cell carcinoma. Clinical, pathological, and genetic features. Am J Pathol 1996;149:1201-8.

15. Eble JN, Amin MB, Young RH. Epithelioid angiomyolipoma of the kidney: a report of five cases with a prominent and diagnostically confusing epithelioid smooth muscle component. Am J Surg Pathol 1997;21:1123-30.

16. Martignoni G, Pea M, Bonetti F, Zamboni G, Carbonara C, Longa L, et al. Carcinoma-like, monotypic epithelioid angiomyolipoma, in patients without evidence of tuberous sclerosis. A clinico-pathologic and genetic study. Am J Surg Pathol 1998;22:663-72.

17. Pea M, Bonetti F, Martignoni G, Henske EP, Manfrin E, Colato C, et al. Apparent renal cell carcinomas in tuberous sclerosis are heterogenous: the identification of malignant epithelioid angiomyolipoma. Am J Surg Pathol 1998;22:180-7.

18. Ruco LP, Pilozzi E, Wedard BM, Marzullo A, D’Andrea V, De Antoni E, et al. Epithelioid lymphangioleiomyomatosis-like tumour of the uterus in a patient without tuberous sclerosis: a lesion mimicking epithelioid leiomyosarcoma (letter). Histopathology 1998;33:91-3.

19. Nonomura A, Minato H, Kurumaya H. Angiomyolipoma predominantly composed of smooth muscle cells: problems in histological diagnosis. Histopathology 1998;33:20-7.

20. Tsui WMS, Colombari R, Portmann BC, Bonetti F, Thung SN, Ferrell LD, et al. Hepatic angiomyolipoma: clinicopathologic study of 30 cases and delineation of unusual morphologic variants. Am J Surg Pathol 1999;23:34-48.

21. Eble JN. Angiomyolipoma of kidney. Semin Diagn Pathol 1998;15:21-40.

22. Martignoni G, Pea M, Rigaud G, Manfrin E, Colato C, Zamboni G, et al. Renal angiomyolipoma with epithelioid sarcomatous transformation and metastases. Demonstration of the same genetic defects in the primary and metastatic lesions. Am J Surg Pathol 2000;24:889-94.

23. Kamishima T, Fukuda T, Emura I, Tanigawa T, Naito M. Pigmented renal cell carcinoma. Am J Surg Pathol 1995;19:350-6.

24. Davis CJ, Sesterhenn IA, Brinsko R, Mostofi FK. Melanocytic clear cell neoplasms of the kidney (abstract). Mod Pathol 1999;12:93A.

25. Ribalta T, Lloreta J, Munne A, Serrano S, Cardesa A. Malignant pigmented clear cell epithelioid tumor of the kidney: clear cell ("sugar") tumor versus malignant melanoma. Hum Pathol 2000;31:516-9.

26. Gomez MR. Phenotypes of the tuberous sclerosis complex with a revision of diagnostic criteria. In: Johnson WG, Gomez MR, editors. Tuberous sclerosis and allied disorders. New York: The New York Academy of Sciences; 1991. p. 1-7.

27. Flieder DB, Travis WD. Clear cell "sugar" tumor of the lung: association with lymphangioleiomyomatosis and multifocal micronodular pneumocyte hyperplasia in a patient with tuberous sclerosis. Am J Surg Pathol 1997;21:1242-7.

28. Martignoni G, Pea M, Bonetti F, Colombari R, Sukhorukov VS, Zamboni G, et al. Clear cell sugar tumor of the lung. A New TS-associated neoplasm [abstract]. Proceedings of the10th World Congress on Tuberous Sclerosis, Goteborg, October 16-17, 1998. p. S-13.

29. Bonetti F, Chiodera PL. Lymphangioleiomyomatosis and tuberous sclerosis: where is the border? Eur Resp J 1996;9:399401.

30. Henske EP, Neumann HPH, Scheithauer BW, Herbst EW, Short MP, Kwiatkowski DJ. Loss of heterozigosity in the tuberous sclerosis (TSC2) region of chromosome band 16p13 occurs in sporadic as well as TSC-associated renal angiomyolipoma. Genes, Chromosom Cancer 1995;13:295-8. 